VOLUME 27 (2020) 88-101

DOI: $10.24330 /$ ieja.662967

\title{
EM-HERMITE RINGS
}

\author{
Emad Abuosba and Manal Ghanem \\ Received: 13 January 2019; Accepted: 5 August 2019 \\ Communicated by Abdullah Harmancı
}

\begin{abstract}
A ring $R$ is called EM-Hermite if for each $a, b \in R$, there exist $a_{1}, b_{1}, d \in R$ such that $a=a_{1} d, b=b_{1} d$ and the ideal $\left(a_{1}, b_{1}\right)$ is regular. We give several characterizations of EM-Hermite rings analogue to those for K-Hermite rings, for example, $R$ is an EM-Hermite ring if and only if any matrix in $M_{n, m}(R)$ can be written as a product of a lower triangular matrix and a regular $m \times m$ matrix. We relate EM-Hermite rings to Armendariz rings, rings with a.c. condition, rings with property A, EM-rings, generalized morphic rings, and PP-rings. We show that for an EM-Hermite ring, the polynomial ring and localizations are also EM-Hermite rings, and show that any regular row can be extended to regular matrix. We relate EM-Hermite rings to weakly semi-Steinitz rings, and characterize the case at which every finitely generated $R$-module with finite free resolution of length 1 is free.
\end{abstract}

Mathematics Subject Classification (2010): 13Axx, 13B25, 13B30, $13 \mathrm{C} 10$ Keywords: Hermite ring, K-Hermite ring, weakly semi-Steinitz ring, generalized morphic ring, regular matrix

\section{Introduction}

All rings are assumed to be commutative with unity 1 . For any ring $R$, let $Z(R)$ be the set of all zero-divisors, and $\operatorname{reg}(R)=R \backslash Z(R)$ be the set of all regular elements, and let $U(R)$ be the set of all units in $R$. Recall that if $R$ is a commutative ring with unity, then the total quotient ring of $R$ is the localization $T(R)=(\operatorname{reg}(R))^{-1} R$. Let $M_{n, m}(R)$ be the ring of all $n \times m$ matrices defined on $R$. It is well known that $A \in U\left(M_{n, n}(R)\right)$ if and only if $\operatorname{det}(A) \in U(R), A \in \operatorname{reg}\left(M_{n, n}(R)\right)$ if and only if $\operatorname{det}(A) \in \operatorname{reg}(R)$, and $A$ is left zero-divisor if and only if it is right zero-divisor, see [3]. The row $\left[\begin{array}{llll}a_{1} & a_{2} & \cdots & a_{n}\end{array}\right]$ is called unimodular if the ideal $\left(a_{1}, a_{2}, \cdots, a_{n}\right)=R$, and it is called regular if the ideal $\left(a_{1}, a_{2}, \cdots, a_{n}\right) \nsubseteq Z(R)$, in this case the ideal $\left(a_{1}, a_{2}, \cdots, a_{n}\right)$ is called a regular ideal. Similar definitions are for columns.

The first author was supported by the "Scientific Research Deanship" at "The University of Jordan". 
A ring $R$ is called a K-Hermite ring if for each $a, b \in R$, there exist $a_{1}, b_{1}, d \in R$ such that $a=a_{1} d, b=b_{1} d$ and the ideal $\left(a_{1}, b_{1}\right)=R$, see [6] and [8]. It is clear that if $R$ is a K-Hermite ring, then it is a Bézout ring (every finitely generated ideal is principal). A ring $R$ is called Hermite if any unimodular row over $R$ can be completed to an invertible matrix by adding a suitable number of new rows. Any K-Hermite is Hermite, but the converse is not true, see [10].

We generalize the concept of K-Hermite rings in the following sense: we call a ring $R$ EM-Hermite, if for each $a, b \in R$, there exist $a_{1}, b_{1}, d \in R$ such that $a=a_{1} d, b=b_{1} d$ and the ideal $\left(a_{1}, b_{1}\right)$ is regular. We find that this ring has some nice properties; it is preserved by the direct products and localizations, and unlike the case of K-Hermite rings, if $R$ is EM-Hermite, then so is $R[x]$. We give several characterizations of EM-Hermite rings analogue to those for K-Hermite rings, for example, $R$ is an EM-Hermite ring if and only if any matrix in $M_{n, m}(R)$ can be written as a product of a lower triangular matrix and a regular $m \times m$ matrix. We also show that any regular row can be extended to a regular matrix by adding a suitable number of rows. We prove that EM-Hermite rings are non-comparable with Bézout rings, nor Hermite rings, but $R$ is K-Hermite if and only if it is Bézout EM-Hermite. We also relate EM-Hermite rings to Armendariz rings, rings with a.c. condition, rings with property A, PP-rings, weakly semi-Steinitz rings, EM-rings, and generalized morphic rings. Finally, we characterize when an $R$-module with finite free resolution of length 1 is free.

\section{EM-Hermite rings}

In this section, we define EM-Hermite rings, and give several characterizations for it, and study some cases at which an EM-Hermite ring is K-Hermite.

Definition 2.1. A ring $R$ is called EM-Hermite if for each $a, b \in R$, there exist $a_{1}, b_{1}, d \in R$ such that $a=a_{1} d, b=b_{1} d$ and the ideal $\left(a_{1}, b_{1}\right)$ is regular.

We now give some examples of EM-Hermite rings.

Example 2.2. (1) Since any principal ideal ring is K-Hermite, see [10], it is also EM-Hermite.

(2) It is clear that any integral domain is an EM-Hermite ring, and so, $\mathbb{Z}[x]$ is an EM-Hermite ring that is not K-Hermite, being non-Bézout.

(3) Consider the idealization $\mathbb{Z}_{4}(+) \mathbb{Z}_{4}$, and consider the two elements $(2,0)$ and $(0,1)$. Assume $(2,0)=(a, b)(c, d)$ and $(0,1)=(a, b)(x, y)$.

If $x \neq 0$, then we must have $a=2=x$, and so we have $1=2 y+2 b$, and hence $2=0$, a contradiction. 
So, we must have $x=0$, and hence, $1=a y$, i.e. $a$ is a unit in $\mathbb{Z}_{4}$. Thus we have $c=2$. Now,

$$
\begin{aligned}
& (2, d)(0,2)=(0,0), \\
& (0, y)(0,2)=(0,0) .
\end{aligned}
$$

Hence $\operatorname{Ann}((2, d),(0, y)) \neq\{(0,0)\}$, and $\mathbb{Z}_{4}(+) \mathbb{Z}_{4}$ is not an EM-Hermite ring. Since any finite ring is Hermite, then $\mathbb{Z}_{4}(+) \mathbb{Z}_{4}$ is Hermite that is not EM-Hermite.

(4) Let $R=\mathbb{Z}\left[x_{1}, x_{2}, x_{3}, y_{1}, y_{2}, y_{3}\right] /\left(x_{1} y_{1}+x_{2} y_{2}+x_{3} y_{3}-1\right)$. Then $R$ is an integral domain, and hence EM-Hermite that is not a Hermite ring, see [12].

We now give equivalent characterizations of EM-Hermite rings, parallel to those for K-Hermite, see [10].

Theorem 2.3. The following statements are equivalent for a ring $R$.

(1) $R$ is an EM-Hermite ring.

(2) For any finite set $\left\{a_{1}, a_{2}, \ldots, a_{n}\right\} \subseteq R$, there exists $\left\{b_{1}, b_{2}, \ldots, b_{n}, d\right\} \subseteq R$ such that $a_{i}=b_{i} d$, for each $i$, and the ideal $\left(b_{1}, b_{2}, \ldots, b_{n}\right)$ is regular.

(3) For any finite set $\left\{a_{1}, a_{2}, \ldots, a_{n}\right\} \subseteq R$, there exist $d \in R$ and a regular matrix $Q \in M_{n, n}(R)$ such that $\left[\begin{array}{llll}a_{1} & a_{2} & \ldots & a_{n}\end{array}\right]=\left[\begin{array}{lllll}d & 0 & 0 & \ldots & 0\end{array}\right] Q$.

(4) For any matrix $B \in M_{m, n}(R)$, there exists a regular matrix $Q \in M_{n, n}(R)$ such that $B=L Q$, with $L$ a lower triangular matrix.

Proof. (1) $\Rightarrow(2)$ Assume $R$ is an EM-Hermite ring, and let $a, b, c \in R$. Then there exist $a_{1}, b_{1}, d \in R$ such that $a=a_{1} d, b=b_{1} d$ and $r_{1}=\alpha_{1} a_{1}+\beta_{1} b_{1} \in$ $\left(a_{1}, b_{1}\right) \cap \operatorname{reg}(R)$. Also there exist $a_{2}, b_{2}, k \in R$ such that $d=a_{2} k, c=b_{2} k$ and $r_{2}=\alpha_{2} a_{2}+\beta_{2} b_{2} \in\left(a_{2}, b_{2}\right) \cap \operatorname{reg}(R)$.

But $a=a_{1} d=a_{1} a_{2} k$ and $b=b_{1} d=b_{1} a_{2} k$. Also we have $r_{1} r_{2}=\left(\alpha_{1} \alpha_{2}\right)\left(a_{1} a_{2}\right)+$ $\left(\alpha_{2} \beta_{1}\right)\left(a_{2} b_{1}\right)+\left(\alpha_{1} \beta_{2} a_{1}+\beta_{1} \beta_{2} b_{1}\right)\left(b_{2}\right) \in\left(b_{2}, a_{1} a_{2}, a_{2} b_{1}\right) \cap r e g(R)$. So, the condition can be applied to any finite subset of $R$.

$(2) \Rightarrow(3)$ Let $\left\{a_{1}, a_{2}, \ldots, a_{n}\right\} \subset R$. Then there exits $\left\{b_{n-1}, b_{n}, d_{1}\right\} \subseteq R$ such that $a_{i}=b_{i} d_{1}$, for $i \in\{n, n-1\}$, and $r_{1}=\alpha_{n-1} b_{n-1}+\alpha_{n} b_{n} \in\left(b_{n-1}, b_{n}\right) \cap \operatorname{reg}(R)$. So we have

$\left[\begin{array}{llll}a_{1} & a_{2} & \ldots & a_{n}\end{array}\right]=\left[\begin{array}{llllll}a_{1} & a_{2} & \ldots & a_{n-2} & d_{1} & 0\end{array}\right] Q_{1}$,

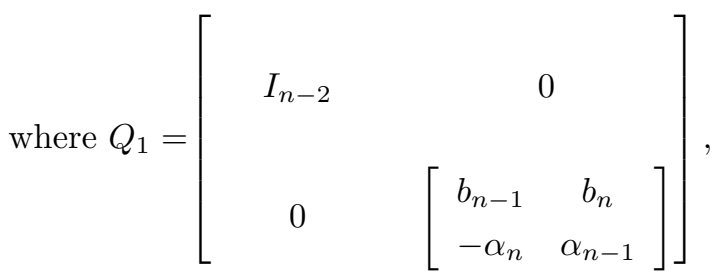
and note that $\operatorname{det}\left(Q_{1}\right)=r_{1} \in \operatorname{reg}(R)$. 
There exists $\left\{b_{n-3}, b_{n-2}, d_{2}\right\} \subset R$ such that $a_{n-2}=b_{n-2} d_{2}, d_{1}=b_{n-3} d_{2}$ and $r_{2}=\alpha_{n-2} b_{n-2}+\alpha_{n-3} b_{n-3} \in\left(b_{n-2}, b_{n-3}\right) R \cap \operatorname{reg}(R)$. So we have

$\left[\begin{array}{llllll}a_{1} & a_{2} & \ldots & a_{n-2} & d_{1} & 0\end{array}\right]=\left[\begin{array}{lllllll}a_{1} & a_{2} & \ldots & a_{n-3} & d_{2} & 0 & 0\end{array}\right] Q_{2}$,

where $Q_{2}=\left[\begin{array}{ccc}I_{n-3} & 0 & \\ 0 & {\left[\begin{array}{ccc}b_{n-2} & b_{n-3} & 0 \\ -\alpha_{n-3} & \alpha_{n-2} & 0 \\ 0 & 0 & 1\end{array}\right]}\end{array}\right]$,

and note that $\operatorname{det}\left(Q_{2}\right)=r_{2} \in \operatorname{reg}(R)$.

In this case we have $\left[\begin{array}{llll}a_{1} & a_{2} & \ldots & a_{n}\end{array}\right]=\left[\begin{array}{lllllll}a_{1} & a_{2} & \ldots & a_{n-3} & d_{2} & 0 & 0\end{array}\right] Q_{2} Q_{1}$, and $\operatorname{det}\left(Q_{2} Q_{1}\right)=r_{2} r_{1} \in \operatorname{reg}(R)$.

Continue to get $\left[\begin{array}{llll}a_{1} & a_{2} & \ldots & a_{n}\end{array}\right]=\left[\begin{array}{lllll}d & 0 & 0 & \ldots & 0\end{array}\right] Q$, and $\operatorname{det}(Q)=r \in \operatorname{reg}(R)$.

$(3) \Rightarrow(4)$ Let $B \in M_{m, n}(R)$. We will proceed by induction on $m$. By (3) the result is true when $m=1$. So assume it is true for all $k<m$, and let $B=\left[b_{i j}\right]_{m \times n}$. It follows by (3) that $\left[\begin{array}{lllll}b_{11} & b_{12} & \ldots & b_{1 n}\end{array}\right]=\left[\begin{array}{lllll}d & 0 & 0 & \ldots & 0\end{array}\right] Q_{1}$, where $Q_{1}$ is a regular matrix. So, $\left[\begin{array}{lllll}b_{11} & b_{12} & \ldots & b_{1 n}\end{array}\right] \operatorname{adj}\left(Q_{1}\right)=\operatorname{det}\left(Q_{1}\right)\left[\begin{array}{lllll}d & 0 & 0 & \ldots & 0\end{array}\right]$. Thus, $B \operatorname{adj}\left(Q_{1}\right)=$ $\operatorname{det}\left(Q_{1}\right)\left[\begin{array}{ll}d & 0 \\ C & D\end{array}\right]$. By induction hypothesis we have $D=L_{1} Q_{2}$, where $L_{1}$ is a lower triangular matrix and $Q_{2}$ is regular matrix in $M_{(n-1),(n-1)}(R)$. Substituting we get

$$
B \operatorname{adj}\left(Q_{1}\right)=\operatorname{det}\left(Q_{1}\right)\left[\begin{array}{cc}
d & 0 \\
C & L_{1} Q_{2}
\end{array}\right]=\operatorname{det}\left(Q_{1}\right)\left[\begin{array}{cc}
d & 0 \\
C & L_{1}
\end{array}\right]\left[\begin{array}{cc}
1 & 0 \\
0 & Q_{2}
\end{array}\right],
$$

and so,

$$
B=\left[\begin{array}{cc}
d & 0 \\
C & L_{1}
\end{array}\right]\left[\begin{array}{cc}
1 & 0 \\
0 & Q_{2}
\end{array}\right] Q_{1}
$$

Now, let $L=\left[\begin{array}{cc}d & 0 \\ C & L_{1}\end{array}\right]$, and $Q=\left[\begin{array}{cc}1 & 0 \\ 0 & Q_{2}\end{array}\right] Q_{1}$. Then $L$ is lower $\operatorname{triangular,~} \operatorname{det}(Q)=$ $\operatorname{det}\left(Q_{2}\right) \operatorname{det}\left(Q_{1}\right) \in \operatorname{reg}(R)$, and $B=L Q$.

$(4) \Rightarrow(1)$ Let $a, b \in R$, Then there exist $d \in R$, and a regular matrix $Q \in M_{2,2}(R)$ such that $[a b]=\left[\begin{array}{ll}a & 0\end{array}\right] Q$.

So, $a=d q_{11}, b=d q_{12}$, and $\operatorname{det}(Q)=q_{11} q_{22}-q_{12} q_{21} \in\left(q_{11}, q_{12}\right) \cap \operatorname{reg}(R)$. Thus, $R$ is an EM-Hermite ring.

If we extend our work to non-commutative rings, we will have:

Corollary 2.4. If $R$ is an EM-Hermite ring, then $M_{n, n}(R)$ is also EM-Hermite. 
Proof. Assume $R$ is an EM-Hermite ring, and let $A, B \in M_{n, n}(R)$. Then there exist lower triangular matrix $L \in M_{n, 2 n}(R)$ and a regular matrix $Q \in M_{2 n, 2 n}(R)$ such that

$$
\left[\begin{array}{ll}
A & B
\end{array}\right]=L Q=\left[\begin{array}{ll}
L_{1} & 0
\end{array}\right]\left[\begin{array}{ll}
Q_{1} & Q_{2} \\
Q_{3} & Q_{4}
\end{array}\right] .
$$

So it follows by (3) in Theorem 2.3 that $M_{n, n}(R)$ is EM-Hermite.

We can follow the proof of [10] to show that the following statements are equivalent.

Proposition 2.5. The following statements are equivalent for a ring $R$.

(1) For any matrix $B \in M_{m, n}(R)$, there exists a regular matrix $Q \in M_{n, n}(R)$ such that $B Q=L$ a lower triangular matrix.

(2) For any vector $\left[\begin{array}{llll}a_{1} & a_{2} & \ldots & a_{n}\end{array}\right] \in M_{1, n}(R)$, there exists a regular matrix $Q \in M_{n, n}(R)$ and $d \in R$ such that $\left[\begin{array}{llll}a_{1} & a_{2} & \ldots & a_{n}\end{array}\right] Q=\left[\begin{array}{lllll}d & 0 & 0 & \ldots & 0\end{array}\right]$.

(3) For any $a, b \in R$, there exists a regular matrix $Q \in M_{2,2}(R)$ and $d \in R$ such that $\left[\begin{array}{ll}a_{1} & a_{2}\end{array}\right] Q=\left[\begin{array}{ll}d & 0\end{array}\right]$.

(4) For any $a, b \in R$, there exist $x, y \in R$ such that $a x+b y=0$ and $(x, y)$ is a regular ideal in $R$.

Assume that $R$ is an EM-Hermite ring, and let $a, b, d,-x, y \in R$ such that $a=d y, b=d(-x)$ and $\beta(-x)+\alpha y=r \in \operatorname{reg}(R)$. Then $a x+b y=0$. So, $R$ satisfies condition (4) in Proposition 2.5, and hence it satisfies all the conditions. Moreover we have:

$$
\begin{aligned}
& {\left[\begin{array}{ll}
a & b
\end{array}\right]=\left[\begin{array}{ll}
d & 0
\end{array}\right]\left[\begin{array}{cc}
y & -x \\
-\beta & \alpha
\end{array}\right],} \\
& {\left[\begin{array}{ll}
a & b
\end{array}\right]\left[\begin{array}{ll}
\alpha & x \\
\beta & y
\end{array}\right]=\left[\begin{array}{ll}
d r & 0
\end{array}\right],}
\end{aligned}
$$

with $\operatorname{det}\left[\begin{array}{cc}y & -x \\ -\beta & \alpha\end{array}\right]=\operatorname{det}\left[\begin{array}{cc}\alpha & x \\ \beta & y\end{array}\right]=r \in \operatorname{reg}(R)$.

To give a more general result, let $B \in M_{m, n}(R)$. There exists a regular matrix $Q \in M_{n, n}(R)$ such that $B=L Q$ with $L$ a lower triangular matrix. Then $B \operatorname{adj}(Q)=\operatorname{det}(Q) L$. Moreover, $\operatorname{det}(Q) L$ is a lower triangular matrix and $\operatorname{det}(\operatorname{adj}(Q))=(\operatorname{det}(Q))^{n-1} \in \operatorname{reg}(R)$.

Although EM-Hermite rings are in general not K-Hermite, the following Theorem shows that for some rings they are equivalent.

Theorem 2.6. If every regular element in $R$ is a unit, then $R$ is a K-Hermite ring if and only if it is an EM-Hermite ring. 
The condition in the above Theorem is not necessary, since $\mathbb{Z}$ has regular elements that are not units, but it is K-Hermite.

Corollary 2.7. If $R$ is a finite ring, then $R$ is K-Hermite ring if and only if it is an EM-Hermite ring.

Corollary 2.8. For any ring $R, T(R)$ is K-Hermite ring if and only if it is an EM-Hermite ring.

We now continue the investigation started in [7], [8] and [11] for the cases at which a Bézout ring is K-Hermite.

Theorem 2.9. A ring $R$ is K-Hermite if and only if it is a Bézout EM-Hermite ring.

Proof. If $R$ is K-Hermite, then clearly it is a Bézout EM-Hermite ring. So assume that $R$ is a Bézout EM-Hermite ring, and let $a, b \in R$. Then there exist $a_{1}, b_{1}, d \in R$ such that $a=a_{1} d, b=b_{1} d$ and $\left(d_{1}\right)=\left(a_{1}, b_{1}\right)$ is a regular ideal in $R$, and so $d_{1} \in \operatorname{reg}(R)$. Thus we have:

$$
\begin{gathered}
d_{1}=a_{1} x+b_{1} y, \\
a_{1}=\alpha d_{1}, \\
b_{1}=\beta d_{1} .
\end{gathered}
$$

Hence we get

$$
d_{1}=d_{1}(\alpha x+\beta y),
$$

and since $d_{1} \in \operatorname{reg}(R)$, we would have

$$
1=\alpha x+\beta y .
$$

Therefore, $a=\alpha\left(d_{1} d\right), b=\beta\left(d_{1} d\right)$ and $(\alpha, \beta)=R$, i.e. $R$ is K-Hermite.

\section{Relations with other rings}

In this section, we relate EM-Hermite rings to Armendariz rings, rings with a.c. condition, rings with property A, EM-rings, generalized morphic rings, and PP-rings.

A ring $R$ is said to be Armendariz if the product of two polynomials in $R[x]$ is zero if and only if the product of their coefficients is zero.

Theorem 3.1. If $R$ is an EM-Hermite ring, then it is Armendariz. 
Proof. Let $f(x)=\sum_{i=0}^{n} f_{i} x^{i}$. Then it follows by Theorem 2.3 that $f_{i}=k_{i} h$ for each $i$ and $\operatorname{Ann}\left(k_{0}, \ldots, k_{n}\right)=\{0\}$. So it follows by McCoy's Theorem that $\sum_{i=0}^{n} k_{i} x^{i}$ is not a zero-divisor in $R[x]$, and $f(x)=h \sum_{i=0}^{n} k_{i} x^{i}$. If $g(x)=\sum_{i=0}^{m} g_{i} x^{i}=k \sum_{i=0}^{m} l_{i} x^{i}$ with $\sum_{i=0}^{m} l_{i} x^{i}$ is not a zero-divisor in $R[x]$. Then $f(x) g(x)=0$ if and only if $h k=0$. Thus we have $f_{i} g_{j}=(h k)\left(k_{i} l_{j}\right)=0$ for each $i$ and $j$. Hence $R$ is Armendariz.

A ring $R$ is said to have a.c. condition, if for any $a, b \in R$ there exists $c \in R$ such that $\operatorname{Ann}(a, b)=\operatorname{Ann}(c)$.

Theorem 3.2. If $R$ is an EM-Hermite ring, then it has a.c. condition.

Proof. Let $a, b \in R$. Then there exist $d, x, y$ such that $a=d x, b=d y$ and the ideal $(x, y)$ is regular. Thus we have $\operatorname{Ann}(x, y)=\{0\}$ and so, $\operatorname{Ann}(a, b)=\operatorname{Ann}(d)$.

A ring $R$ is said to have property $\mathrm{A}$, if any finitely generated ideal contained in $Z(R)$ has nonzero annihilator. It was shown in [9] that any Noetherian ring has property A, see Theorem 82 .

Theorem 3.3. If $R$ is an EM-Hermite ring, then it has property $A$.

Proof. Let $a, b \in R$ such that $\operatorname{Ann}(a, b)=\{0\}$. Then there exist $d, x, y$ such that $a=d x, b=d y$ and the ideal $(x, y)$ is regular. Let $r=\alpha x+\beta y \in \operatorname{reg}(R)$. But $d \in \operatorname{reg}(R)$ since $\operatorname{Ann}(d)=\operatorname{Ann}(a, b)=\{0\}$. Thus we have

$$
a \alpha+b \beta=d x \alpha+d y \beta=d r \in(a, b) \cap \operatorname{reg}(R) .
$$

Therefore, $(a, b) \nsubseteq Z(R)$.

Let $R$ be a ring, and let $f(x) \in Z(R[x])$ such that $f(x)=c_{f} f_{1}(x)$, where $c_{f} \in R$ and $f_{1}(x) \in \operatorname{reg}(R[x])$. Then $c_{f}$ is called an annihilating content for $f(x)$. It is clear that $\operatorname{deg}(f) \leq \operatorname{deg}\left(f_{1}\right)$. If every zero-divisor polynomial in $R[x]$ has an annihilating content, $R$ is called an EM-ring. A ring $R$ is called generalized morphic ring if $\operatorname{Ann}(a)$ is a principal ideal for each $a \in R$, see [1]. Using Theorem 2.3, one can see easily that any EM-Hermite ring is an EM-ring. But the following Theorem shows that the two properties are equivalent if the ring was Noetherian. But first we need the following important lemma.

Lemma 3.4 ([1, Lemma 3.25]). Assume that $R$ is a Noetherian ring, and $b R$ is a prime principal ideal with $b \in Z(R)$. If $a \in b R \backslash\{0\}$, then $a=b^{n}$ s for some $n \in \mathbb{N}$ and $s \in R \backslash b R$. 
Theorem 3.5. Assume that $R$ is a Noetherian ring. Then the following are equivalent:

(1) $R$ is an EM-ring.

(2) $R$ is a generalized morphic ring.

(3) $R$ is an EM-Hermite ring.

Proof. For the equivalence of (1) and (2), see [1].

$(2) \Rightarrow(3)$ Recall first that since $R$ is a Noetherian ring, then $\operatorname{Ann}\left(a_{1}, a_{2}\right) \neq\{0\}$ if and only if the ideal $\left(a_{1}, a_{2}\right) \subseteq Z(R)$.

Let $a_{1}, a_{2} \in R$. If $\operatorname{Ann}\left(a_{1}, a_{2}\right)=\{0\}$, then $a_{1}=a_{1} .1, a_{2}=a_{2} .1$, and $\operatorname{Ann}\left(a_{1}, a_{2}\right)=$ $\{0\}$. If $0 \neq m \in \operatorname{Ann}\left(a_{1}, a_{2}\right)$, then $\left(a_{1}, a_{2}\right) \subseteq \operatorname{Ann}(m) \subseteq M_{1}=c_{1} R \subseteq Z(R)$, where $M_{1}$ is a maximal ideal in $Z(R)$, and so it is prime, see [9, Theorem 6]. Hence, using Lemma 3.4, $a_{i}=\alpha_{i} c_{1}^{k_{i}}$ with $\alpha_{i} \notin c_{1} R$, and $k_{i} \geq 1$ for each $i=1,2$. Let $k_{11}=\operatorname{Min}\left\{k_{i}\right\}, b_{i}=\alpha_{i} c_{1}^{k_{i}-k_{11}}$. Then $a_{i}=c_{1}^{k_{11}} b_{i}$ and $\left(a_{1}, a_{2}\right) \subset\left(b_{1}, b_{2}\right)$. Then repeat the work to write $b_{i}=c_{2}^{k_{22}} d_{i}$ and $\left(a_{1}, a_{2}\right) \subset\left(b_{1}, b_{2}\right) \subset\left(d_{1}, d_{2}\right)$. Continue to get an ascending chain in the Noetherian ring $R$, and thus it must terminate. Hence there exits $f_{i} \in R$ and $a_{i}=c_{1}^{k_{11}} c_{2}^{k_{22}} c_{3}^{k_{33}} \ldots n_{n}^{k_{n n}} f_{i}=c f_{i}$ with $\operatorname{Ann}\left(f_{1}, f_{2}\right)=\{0\}$.

$(3) \Rightarrow(1)$ Clear.

It was shown in [5] that if $X=\beta \mathbb{R}^{+}-\mathbb{R}^{+}$, then $C(X)$ is a K-Hermite, and hence EM-Hermite ring, and since $X$ is connected, $C(X)$ is not generalized morphic ring. Also it was shown in [5] that if $X=[-1,1] \times[0, \infty)$, then $C(\beta X-X)$ is a Bézout ring that is not K-Hermite, then it follows by Theorem 2.9 that $C(\beta X-X)$ is not an EM-Hermite ring. Also it follows by [1] that $C(\beta X-X)$ is an EM-ring.

We note that the Bézout property and the EM-Hermite property are non-comparable, but adding them together would give the K-Hermite property, unlike the case of Hermite property and the EM-Hermite property, they are noncomparable, and adding them together need not be K-Hermite as in the case of $\mathbb{Z}[x]$.

Recall that a ring $R$ is called a PP-ring if every principal ideal in $R$ is a projective $R$-module. While any von Neumann regular ring is K-Hermite, $\mathbb{Z}[x]$ is a PP-ring that is not K-Hermite.

Theorem 3.6. If $R$ is a PP-ring, then it is an EM-Hermite ring.

Proof. Let $a_{1}, a_{2} \in R$. Then $a_{i}=u_{i} e_{i}$, where $u_{i} \in \operatorname{reg}(R)$ and $e_{i}$ is an idempotent for each $i$, see [4, Lemma 2]. Let $e=e_{1}+e_{2}-e_{1} e_{2}$. Then $e$ is also an idempotent and $e_{i} e=e_{i}$ for $i=1,2$. Thus $a_{i}=e u_{i}\left(e_{i}+1-e\right)$, and since $1=\left(e_{1}+1-e\right)+\left(e_{2}+1-\right.$ $e)-\left(e_{1}+1-e\right)\left(e_{2}+1-e\right)$, we have $u_{1} u_{2}=\left(u_{1}\left(e_{1}+1-e\right)\right) u_{2}+\left(u_{2}\left(e_{2}+1-e\right)\right) u_{1}-$ $u_{1}\left(e_{1}+1-e\right) u_{2}\left(e_{2}+1-e\right) \in\left(u_{1}\left(e_{1}+1-e\right), u_{2}\left(e_{2}+1-e\right)\right) \cap \operatorname{reg}(R)$. 
The converse of this theorem needs not be true, since $\mathbb{Z}_{8}$ is an EM-Hermite ring which is not a PP-ring, being non-reduced.

\section{Some properties of EM-Hermite rings}

In this section, we study some properties of EM-Hermite rings, such as polynomial rings and localizations of EM-Hermite rings, and extending regular rows to regular matrices.

The ring $\mathbb{Z}$ is K-Hermite, but $\mathbb{Z}[x]$ is not, and it is conjectured that if $R$ is Hermite, then $R[x]$ is Hermite. We now show that if $R$ is an EM-Hermite ring, then $R[x]$ is EM-Hermite.

Theorem 4.1. If $R$ is an EM-Hermite ring, then $R[x]$ is an EM-Hermite ring.

Proof. Let $f(x)=\sum_{i=0}^{n} f_{i} x^{i}, g(x)=\sum_{i=0}^{m} g_{i} x^{i} \in R[x]$. Then it follows by Theorem 2.3 that $f_{i}=k_{i} h, g_{i}=l_{i} h$, for each $i$ and the ideal $\left(k_{0}, \ldots, k_{n}, l_{0}, \ldots, l_{m}\right) \nsubseteq Z(R)$. Thus, $f(x)=h \sum_{i=0}^{n} k_{i} x^{i}, g(x)=h \sum_{i=0}^{m} l_{i} x^{i}$. If $\sum_{i=0}^{l} h_{i} x^{i} \in \operatorname{Ann}\left(\sum_{i=0}^{n} k_{i} x, \sum_{i=0}^{m} l_{i} x^{i}\right)$, then since $R$ is Armendariz, $h_{i} \in \operatorname{Ann}\left(k_{0}, \ldots, k_{n}, l_{0}, \ldots, l_{m}\right)=\{0\}$ for each $i$, and so, $A n n\left(\sum_{i=0}^{n} k_{i} x, \sum_{i=0}^{m} l_{i} x^{i}\right)=\{0\}$, and since $R[x]$ has property A for any ring $R$, see [9], we have $\left(\sum_{i=0}^{n} k_{i} x, \sum_{i=0}^{m} l_{i} x^{i}\right) R[x] \nsubseteq \nsubseteq Z(R[x])$.

Corollary 4.2. Let $R$ be an EM-Hermite ring. Then $R\left[x_{1}, x_{2}, \ldots, x_{n}\right]$ is an EMHermite ring.

Theorem 4.3. Let $R$ be an EM-Hermite ring, and let $S$ be a multiplicatively closed subset of $R$. Then $S^{-1} R$ is an EM-Hermite ring.

Proof. Let $a, b \in S^{-1} R$. Then there exist $t, s \in S$ such that $t a, s b \in R$. Since $R$ is an EM-Hermite ring, there exist $d, a_{1}, b_{1} \in R$ such that $t a=d a_{1}$ and $s b=d b_{1}$ and $\left(a_{1}, b_{1}\right)$ is a regular ideal in $R$. There exist $x, y \in R$ such that $r=x a_{1}+y b_{1} \in$ $\operatorname{reg}(R)$. Thus we have $a=d\left(\frac{a_{1}}{t}\right)$ and $b=d\left(\frac{b_{1}}{s}\right)$.

Now, $\frac{x}{s} \frac{a_{1}}{t}+\frac{y}{t} \frac{b_{1}}{s}=\frac{r}{s t} \in\left(\frac{a_{1}}{t}, \frac{b_{1}}{s}\right) \cap \operatorname{reg}\left(S^{-1} R\right)$.

Corollary 4.4. Let $R$ be an EM-Hermite ring. Then $T(R)$ is K-Hermite.

The converse of this Corollary is not in general true as illustrated in the following example.

Example 4.5. It was shown in [1] that if $R=\mathbb{Z}_{6}[x, y] /(x y)$, then $T(R)$ is a von Neumann regular ring, and hence it is K-Hermite. But $R$ is not an EM-Hermite ring, since $x, 3 \in R$, and if $x=a h, 3=b h$ with $\operatorname{Ann}(a, b)=\{0\}$, then $0=a(2 y h)=$ 
$b(2 y h)$, which implies that $0=2 y h$, and so, $(h) \subseteq A n n(2 y)=(3, x) \subseteq(h)$, and so, $(h)=(3, x)$, a contradiction.

Theorem 4.6. If $R$ is an EM-Hermite ring, then any regular row can be completed to a regular square matrix by adding a suitable number of rows.

Proof. We will proceed by induction on $n$, and make some modifications on the proof of [10, page 28].

If $n=2$, and $\left[\begin{array}{ll}a_{1} & a_{2}\end{array}\right]$ is regular, then $a_{1} t+a_{2} s=r \in \operatorname{reg}(R)$, and $\operatorname{det}\left[\begin{array}{cc}a_{1} & a_{2} \\ -s & t\end{array}\right]=r \in \operatorname{reg}(R)$. So, assume that the result is true for all $m<n$, and consider the regular row $\left[\begin{array}{llll}a_{1} & a_{2} & \cdots & a_{n}\end{array}\right]$. Since $R$ is an EM-Hermite ring, $a_{i}=d c_{i}, 1 \leq i<n$, and $\left(c_{1}, c_{2}, \cdots, c_{n-1}\right) \nsubseteq Z(R)$, and so, the regular row $\left[\begin{array}{llll}c_{1} & c_{2} & \cdots & c_{n-1}\end{array}\right]$ can be extended to an $(n-1) \times(n-1)$ regular matrix $C$. Again, since $R$ is an EM-Hermite ring, $a_{n}=k \alpha, d=k \beta$, with $\alpha t+\beta s=r \in \operatorname{reg}(R)$. Note that if $w k=0$, then $w \in \operatorname{Ann}\left(a_{1}, a_{2}, \cdots, a_{n}\right)=\{0\}$, and hence we have $k \in \operatorname{reg}(R)$. Thus $a_{n} t+d s=k \alpha t+k \beta s=k r \in \operatorname{reg}(R)$. Now consider the matrix,

$$
B=\left[\begin{array}{ccc}
d & 0 & a_{n} \\
0 & I_{n-2} & 0 \\
-t & 0 & s
\end{array}\right] .
$$

Then $\operatorname{det}(B)=k r \in \operatorname{reg}(R)$, and the $n \times n$ matrix

$$
A=B\left[\begin{array}{ll}
C & 0 \\
0 & 1
\end{array}\right]
$$

is regular and has first row $\left[\begin{array}{llll}a_{1} & a_{2} & \cdots & a_{n}\end{array}\right]$.

Corollary 4.7. If $R$ is an EM-Hermite ring, then any regular column can be completed to a regular square matrix by adding a suitable number of columns.

Proof. Just take transpose, and the result follows immediately by the previous Theorem.

Corollary 4.8. If $R$ is an EM-Hermite ring, then any unimodular row can be completed to a regular square matrix by adding a suitable number of rows.

Note that in the ring $\mathbb{Z}_{4}(+) \mathbb{Z}_{4}$ any regular row is extendable to a regular matrix, being a finite Hermite ring, although it is not an EM-Hermite ring. 


\section{Applications to finitely presented modules}

In this section, we relate EM-Hermite rings to weakly semi-Steinitz rings, and characterize the case at which every finitely generated $R$-module with finite free resolution of length 1 is free.

An $R$-module $M$ satisfies property $\mathrm{P}$ if any two maximal independent subsets of $M$ have the same cardinality. It was shown in [2] that every free $R$-module satisfies property $\mathrm{P}$ if and only if whenever $a_{1}, \ldots, a_{n} \in R$ such that $A n n_{R}\left(a_{1}, \ldots, a_{n}\right)=$ $\{0\}$, then the row $\left[\begin{array}{llll}a_{1} & a_{2} & \cdots & a_{n}\end{array}\right]$ can be completed to a square regular matrix.

A ring $R$ is called a weakly semi-Steinitz ring if every finite independent subset of a finitely generated free $R$-module can be extended to a basis. The following two propositions characterize weakly semi-Steinitz rings, see [2] and [12].

Proposition 5.1. The following statements are equivalent:

(1) $R$ is a weakly semi-Steinitz ring.

(2) $R$ is Hermite and every finitely generated proper ideal of $R$ has non-zero annihilator.

(3) Every finitely generated proper ideal of $R$ has non-zero annihilator and any finitely generated stably free $R$-module is a direct sum of cyclic modules.

(4) For each $n \geq 1$, every linearly independent element of $R^{n}$ can be extended to a basis of $R^{n}$.

(5) $\operatorname{reg}(R)=U(R)$ and every free $R$-module satisfies property $P$.

Proposition 5.2. Let $R$ be a Noetherian ring. Then $R$ is a weakly semi-Steinitz ring if and only if reg $(R)=U(R)$. If in addition, $R$ is reduced, then $R$ is a weakly semi-Steinitz ring if and only if $R$ is a finite direct product of fields.

We now give extra two characterizations of weakly semi-Steinitz rings.

Theorem 5.3. $R$ is a weakly semi-Steinitz ring if and only if whenever $a_{1}, \ldots, a_{n} \in$ $R$ such that $A n n_{R}\left(a_{1}, \ldots, a_{n}\right)=\{0\}$, then the row $\left[\begin{array}{llll}a_{1} & a_{2} & \cdots & a_{n}\end{array}\right]$ can be completed to a square invertible matrix.

Proof. Assume $R$ is a weakly semi-Steinitz ring and assume that $a_{1}, \ldots, a_{n} \in$ $R$ such that $\operatorname{Ann}_{R}\left(a_{1}, \ldots, a_{n}\right)=\{0\}$. Then $\bar{x}_{1}=\left(a_{1}, \ldots, a_{n}\right) \in R^{n}$ is linearly independent, and so $R^{n}$ has a basis $\left\{\bar{x}_{1}, \ldots, \bar{x}_{n}\right\}$. Let $A=\left[\begin{array}{c}\bar{x}_{1} \\ \vdots \\ \bar{x}_{n}\end{array}\right]$. There exist $c_{i j} \in R$ such that $\sum_{j=1}^{n} c_{i j} \bar{x}_{j}=\bar{e}_{i}$ for $i=1,2, \ldots, n$, where $\left\{\bar{e}_{1}, \ldots, \bar{e}_{n}\right\}$ is the 
standard basis for $R^{n}$. Let $C=\left[c_{i j}\right]$. Then $C A=I_{n}$. Thus, $A$ is a regular matrix, with the ideal $(\operatorname{det}(A))$ is non-proper. Thus $A$ is invertible.

Conversely, it is clear that $R$ is Hermite. Assume that $a_{1}, \ldots, a_{n} \in R$ such that $\operatorname{Ann}_{R}\left(a_{1}, \ldots, a_{n}\right)=\{0\}$. Let $\bar{x}=\left[\begin{array}{llll}a_{1} & a_{2} & \cdots & a_{n}\end{array}\right]$. Then there exists an invertible $n \times n$ matrix $A=\left[\begin{array}{c}\bar{x}_{1} \\ \vdots \\ \bar{x}_{n}\end{array}\right]$. But $\operatorname{det}(A) \in \sum_{i=1}^{n} a_{i} R \cap U(R)$. Thus, the ideal $\left(a_{1}, \ldots, a_{n}\right)$ is non-proper, and $R$ is a weakly semi-Steinitz ring.

Recall that a finitely generated $R$-module $P$ is said to have finite free resolution of length 1 if we have the short exact sequence

$$
0 \longrightarrow R^{m} \stackrel{\alpha}{\longrightarrow} R^{n} \longrightarrow P \longrightarrow 0 .
$$

If the sequence splits, then $P$ is a finitely generated stably free module.

Theorem 5.4. $R$ is a weakly semi-Steinitz ring if and only if every finitely generated $R$-module with finite free resolution of length 1 is free.

Proof. Assume that $R$ is a weakly semi-Steinitz ring, and consider the short exact sequence

$$
0 \longrightarrow R^{m} \stackrel{\alpha}{\longrightarrow} R^{n} \longrightarrow P \longrightarrow 0 .
$$

If $\left\{\bar{a}_{i}\right\}_{i=1}^{m}$ is a basis for $R^{m}$, then $\left\{\alpha\left(\bar{a}_{i}\right)\right\}_{i=1}^{m}$ is a linear independent subset of the weakly semi-Steinitz ring $R^{n}$ and so it can be extended to a basis $\left\{\alpha\left(\bar{a}_{i}\right)\right\}_{i=1}^{m} \cup$ $\left\{\bar{b}_{i}\right\}_{i=1}^{n-m}$. Now, define the $R$-module homomorphism $T: R^{n} \longrightarrow R^{m}$ such that $T\left(\alpha\left(\bar{a}_{i}\right)\right)=\bar{a}_{i}$, and $T\left(\bar{b}_{i}\right)=0$. Then $T \circ \alpha=I d_{R^{m}}$, and so, the exact sequence splits. Thus $P$ is a finitely generated stably free $R$-module, and hence it is free, since $R$ is Hermite.

Conversely, it is clear that $R$ is Hermite. Assume $a_{1}, \ldots, a_{n} \in R$ such that $A n n_{R}\left(a_{1}, \ldots, a_{n}\right)=\{0\}$. Then $\bar{x}=\left(a_{1}, \ldots, a_{n}\right) \in R^{n}$ is linearly independent, and so $\alpha: R \longrightarrow R^{n}$ defined by $\alpha(r)=r \bar{x}$ is an injective $R$-homomorphism. Thus the sequence

$$
0 \longrightarrow R \stackrel{\alpha}{\longrightarrow} R^{n} \longrightarrow R^{n} / \operatorname{Im} \alpha \longrightarrow 0
$$

is short exact, and so, $R^{n} / \operatorname{Im} \alpha$ is a free $R$-module. Thus there exists an $R$ homomorphism $\beta: R^{n} \longrightarrow R$ such that $\beta \circ \alpha=I d_{R}$, and so, $\beta \circ \alpha(1)=1$, and hence

$$
1=M(\beta) M(\alpha)(1)=\left[\begin{array}{lll}
\beta_{1} & \cdots & \beta_{n}
\end{array}\right]\left[\begin{array}{c}
a_{1} \\
\vdots \\
a_{n}
\end{array}\right](1)
$$


where $M(\beta)$ and $M(\alpha)$ are the corresponding matrices for $\beta$ and $\alpha$ respectively. Therefore, the ideal $\left(a_{1}, \ldots, a_{n}\right)$ is non-proper.

Thus $R$ is a weakly semi-Steinitz ring.

It follows by Theorem 4.6 that if $R$ is an EM-Hermite ring, then every free $R$-module satisfies property $\mathrm{P}$. Thus we have the following result:

Theorem 5.5. If $R$ is an EM-Hermite ring, then $T(R)$ is a weakly semi-Steinitz ring.

It is clear that $\mathbb{Z}_{4}(+) \mathbb{Z}_{4}$ is a weakly semi-Steinitz ring that is not EM-Hermite, while $\mathbb{Z}$ is a K-Hermite ring that has $\mathbb{Z}$-modules of finite resolution that are not free.

\section{References}

[1] E. Abuosba and M. Ghanem, Annihilating content in polynomial and power series rings, J. Korean Math. Soc., 56(5) (2019), 1403-1418.

[2] A. Bouanane and F. Kourki, On weakly semi-Steinitz rings, Commutative Ring Theory, Lecture Notes in Pure and Appl. Math., Dekker, New York, 185 (1997), 131-139.

[3] W. C. Brown, Matrices over Commutative Rings, Monographs and Textbooks in Pure and Applied Mathematics, 169, Marcel Dekker, Inc., New York, 1993.

[4] S. Endo, Note on p.p. rings, A supplement to Hattori's paper, Nagoya Math. J., 17 (1960), 167-170.

[5] L. Gillman and M. Henriksen, Rings of continuous functions in which every finitely generated ideal is principal, Trans. Amer. Math. Soc., 82 (1956), 366391.

[6] L. Gillman and M. Henriksen, Some remarks about elementary divisor rings, Trans. Amer. Math. Soc., 82 (1956), 362-365.

[7] M. Henriksen, Some remarks on elementary divisor rings II, Michigan Math. J., 3(2) (1955), 159-163.

[8] I. Kaplansky, Elementary divisors and modules, Trans. Amer. Math. Soc., 66 (1949), 464-491.

[9] I. Kaplansky, Commutative Rings, Revised Edition, The University of Chicago Press, Chicago, 1974.

[10] T. Y. Lam, Serre's Problem on Projective Modules, Springer Monographs in Mathematics, Springer-Verlag, Berlin, 2006. 
[11] M. D. Larsen, W. J. Lewis and T. S. Shores, Elementary divisor rings and finitely presented modules, Trans. Amer. Math. Soc., 187 (1974), 231-248.

[12] B. Nashier and W. Nichols, On Steinitz properties, Arch. Math. (Basel), 57(3) (1991), 247-253.

Emad Abuosba (Corresponding Author) and Manal Ghanem

Department of Mathematics

School of Science

The University of Jordan

Amman, Jordan

e-mails: eabuosba@ju.edu.jo (E. Abuosba)

m.ghanem@ju.edu.jo (M. Ghanem) 